\title{
Comparing clinicopathological features and prognosis of primary pulmonary invasive mucinous adenocarcinoma based on computed tomography findings
}

\author{
Kai $\mathrm{Nie}^{1 \dagger}$, Wei Nie $^{3 \dagger}, \mathrm{Yu}-\mathrm{Xuan}$ Zhang $^{4 \dagger}$ and Hong Yu${ }^{2^{*}}$
}

\begin{abstract}
Objective: To evaluate the relationship between clinicopathologic characteristics and prognosis in patients with invasive mucinous adenocarcinoma (IMA) of the lung.

Methods: A total of 68 patients who underwent surgical resection for primary lung IMA were reviewed during the period of 2009 and 2017. Tumors were classified as solitary-type or pneumonic-type according to the computed tomography (CT) findings. Cox proportional hazards model was used to assess the effects of clinicopathological characteristics on univariate and multivariable analyses of disease-free survival (DFS).

Results: Solitary-type was found in 54 patients, while pneumonic-type was found in 14 patients. The patients' age varied between 56 and 68 years (patients' median age was 61 years). Besides, 50 patients had T1/T2 tumor stage (73.5\%). Compared with solitary-type, higher T stage, $\mathrm{N}$ stage, and pathological stage $(P<0.001)$ were found in pneumonic-type. Moreover, the survival analysis showed that the pneumonic-type had a significantly poorer DFS compared with solitary-type $(P=0.004)$. Univariate analysis showed that pneumonic pattern on $C T$ scan, T stage, pathologic stage, and thyroid transcription factor-1 (TTF-1) were significant predictive factors of survival $(P=0.011$, $0.014,0.013,0.029$, respectively). Multivariate analysis further indicated that pneumonic-type was the only independent prognostic factor for poor survival [hazard ratio $(\mathrm{HR})=6.764,95 \%$ confidence interval $(\mathrm{Cl}): 1.563-$ 29.269, $P=0.011]$.
\end{abstract}

Conclusions: Based on CT findings, the solitary-type IMA is associated with a lower stage and better prognosis compared with the pneumonic-type IMA.

Keywords: Lung cancer, Invasive mucinous adenocarcinoma, Computed tomography, Disease-free survival, Prognosis

\section{Background}

Lung cancer is a major cause of cancer-related deaths worldwide. Non-small cell lung cancer (NSCLC) accounts for more than $80 \%$ of all lung cancers, among which adenocarcinoma is the most common pathological subtype. Invasive mucinous adenocarcinoma (IMA), formerly known as mucinous bronchioloalveolar

\footnotetext{
*Correspondence: yuhongphd@163.com; yuhongphd@126.com

${ }^{\dagger}$ Kai Nie, Wei Nie and Yu-Xuan Zhang contributed equally to this work and were co-first authors.

${ }^{2}$ Department of Radiology, Shanghai Chest Hospital, Shanghai Jiaotong University, No.241 Huaihai West Road, Xuhui Area, Shanghai 200030, China Full list of author information is available at the end of the article
}

carcinoma (BAC), is relatively rare adenocarcinoma, accounting for only $2-5 \%$ of lung invasive adenocarcinomas [1]. According to the International Association for the Study of Lung Cancer (IASLC)/American Thoracic Society (ATS)/European Respiratory Society (ERS) classification system for lung adenocarcinoma published in 2011, due to unique pathological, radiological, and prognostic characteristics, this type of disease has been separated from the adenocarcinomas formerly classified as nonmucinous BAC [2]. In 2015, the World Health Organization (WHO) also classified IMA as an invasive adenocarcinoma subtype [3].

(c) The Author(s). 2019 Open Access This article is distributed under the terms of the Creative Commons Attribution 4.0 International License (http://creativecommons.org/licenses/by/4.0/), which permits unrestricted use, distribution, and 
As a separate category of lung adenocarcinoma, IMA is diagnosed based on tall columnar cell morphology with abundant intracellular or extracellular mucus and with invasive adenocarcinoma patterns, such as lepidic, acinar, papillary, micropapillary, and solid predominant patterns. Based on the percentage of mucinous pattern, IMA can be divided into two groups: pure mucinous (> $90 \%$ invasive mucinous pattern, lepidic predominant pattern) and mixed mucinous/nonmucinous ( $\geqq 10 \%$ of nonmucinous invasive component) [1]. Several studies have demonstrated that the new lung adenocarcinoma classification has a clear prognostic significance [4-6], suggesting that IMAs are typically associated with poor survival outcomes compared with non-mucinous lepidic, acinar, and papillary predominant subtypes of lung adenocarcinoma [7, 8]. Lymph node involvement and distant metastasis are less frequent in IMA compared with other subtypes of adenocarcinoma. Nevertheless, the aerogenous spread and satellite tumors surrounding the main lesion frequently occur in patients with IMA [9]. In addition, a number of studies [10-12] have discussed different groups of IMA based on computed tomography $(\mathrm{CT})$, in which their results indicated that these groups were closely associated with the clinical outcomes. However, due to low incidence of this subtype, the number of patients included in those studies was relatively small, and the patients with pathological diagnosis of IMA were not differentiated from pure mucinous or mixed mucinous/nonmucinous IMA. Therefore, the present study investigated the association between clinicopathological features and prognosis of subtypes of lung primary IMA based on CT findings.

\section{Patients and methods}

This retrospective study was approved by the Institutional Review Board of Shanghai Changzheng Hospital (Shanghai, China), and the requirement for informed consent was waived.

\section{Patients' selection}

A total of 730 patients who underwent surgical resection for lung adenocarcinoma at our hospital between October 2009 and August 2017 were recruited in this study. Inclusion criteria were: 1) patients with mucinous adenocarcinoma confirmed on surgical resection, in which baseline CT examination of chest was performed before surgical resection in our institution; 2) clinical and imaging data available in the electronic medical records. These patients did not undergo radiotherapy or chemotherapy prior surgery. Exclusion criteria were as follows: 1) patients with multiple primary lung cancer; 2) patients with history of other malignancies; 3) patients who diagnosed with new subtypes of mucinous adenocarcinoma in situ (m-AIS) or mucinous minimally invasive adenocarcinoma (m-MIA); 4) patients with mixed invasive mucinous/nonmucinous adenocarcinoma (those patients had $\geqq 10 \%$ of nonmucinous components and had worse survival outcome compared with those with pure IMA or non-mucinous adenocarcinomas) [13, 14]. For patients who diagnosed with BAC or mucinous (colloid) carcinoma according to the 2004 lung adenocarcinoma classification system released by the WHO, all the slides of the resected specimens were reinspected by two experienced lung pathologists.

All included tumors were pathologically confirmed as pure invasive mucinous adenocarcinoma according to the 2015 lung adenocarcinoma classification system released by the WHO. Three additional patients with clinical stage IV IMA were excluded because as were treated with chemotherapy rather than curative surgery due to the multiple extrathoracic metastases. Ultimately, 68 patients with surgically resected primary lung pure IMA were included in the present study.

\section{Evaluation of $\mathrm{CT}$ findings}

All data were obtained using multi-detector CT scanner (Aquilion 16, Toshiba, Japan or Brilliance 256, Philips Healthcare, The Netherlands) in the craniocaudal direction during inspiration by using the following scanning parameters: X-ray tube voltage, $120 \mathrm{kVp}$; automatic tube currents; rotation speed, $0.5 \mathrm{~s}$. CT slice thickness was as follows: $0.625 \mathrm{~mm}$ (6 patients, $8.9 \%), 1 \mathrm{~mm}$ (28 patients, $41.1 \%$ ), and $5 \mathrm{~mm}$ (34 patients, 50\%). Ioversol was used as a contrast medium at a flow rate of $3 \mathrm{ml} / \mathrm{s}$ through the elbow median vein at a dose of $1.5 \mathrm{ml} / \mathrm{kg}$. Vascular phase and parenchymal phase images were obtained at 20 25 and 75 90 s, respectively. Two radiologists (with more than 15 years of experience on imaging of thoracic disease) independently assessed the CT findings based on tumor morphology on the mediastinal (window level, 40 Hounsfield units; width, 400 Hounsfield units) and pulmonary window level settings (window level, -600 Hounsfield units; width, 1,600 Hounsfield units). Consensus was achieved to make a final decision when disagreement in classification occurred between radiologists. Based on preoperative CT data, 68 IMAs were classified into two groups: 1): solitary-type, which was defined as solitary nodule or mass (Fig. 1 a-c); 2) pneumonic-type, that was defined as consolidation without definable shape and distributed along the lung lobe or lung segment, and sometimes with air bronchogram (Fig. $1 \mathrm{~d}-\mathrm{f}$ ) [11]. Other CT characteristics, such as site and number of involved lobes and tumor size were retrospectively assessed as well.

\section{Pathologic analysis}

Pathologic diagnoses were carried out based on the 2011 IASLC/ATS/ERS classification system for lung adenocarcinoma. According to the 2015 WHO criteria, tumors 


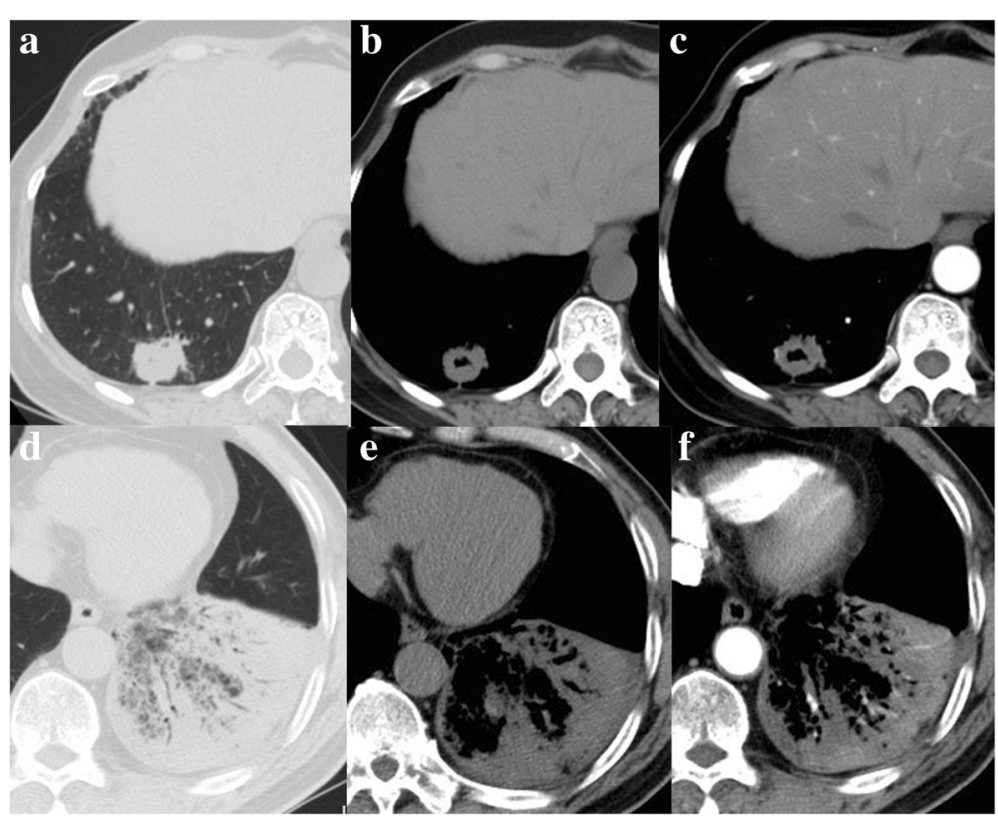

Fig. 1 a-c A53-year-old female who presented solitary-type IMA on plain and contrast-enhanced computed tomography scans. d-f A73-year-old male who presented pneumonic-type IMA on plain and contrast-enhanced computed tomography scans

with $\geqq 90 \%$ mucinous cells were defined as pure IMA. The surgically resected specimens were formalin-fixed, paraffin-embedded, and stained with hematoxylin and eosin (H\&E). All slides of the surgically resected specimens were interpreted by two experienced lung pathologists based on the consensus at the diagnosis of IMA. All slides representing the maximal surface area of the tumor in each patient were reviewed. The tall columnar cells with abundant intracellular mucus represent IMA (Fig. 2). The stages of all IMAs were evaluated based on the 8th edition of the tumor-node-metastasis (TNM) staging classification published by the Union for

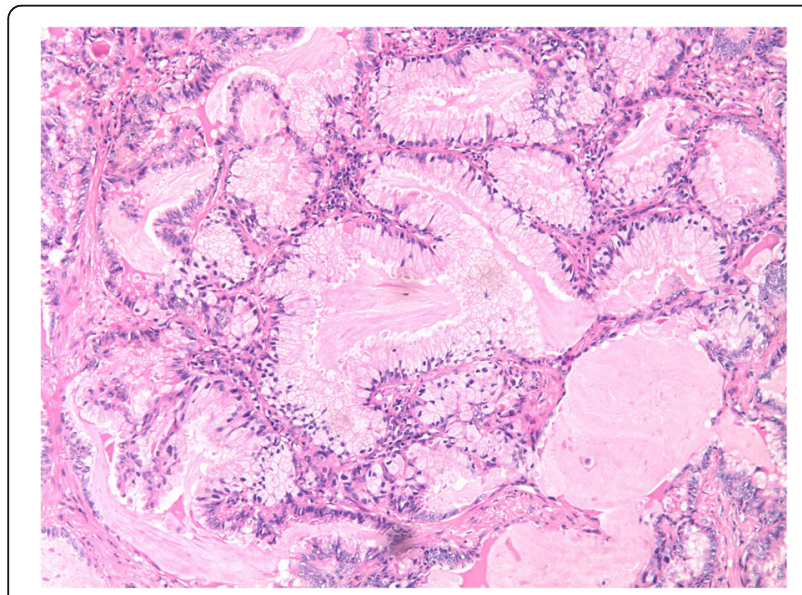

Fig. 2 Histologic specimen showing tall columnar cells with basalnuclei and rich intracellular mucus (hematoxylin and eosin stain; original magnification $\times 100)$
International Cancer Control (UICC) and the American Joint Committee on Cancer (AJCC) [15]. Markers of pulmonary differentiation were observed on one slice of tumor tissue per case using immunohistochemistry analysis. The main makers included nuclear staining for thyroid transcription factor-1 (TTF-1), cytoplasmic staining for cytokeratin 7 (CK7), and cytokeratin 20 (CK20), and positive expression was identified as staining of the cytoplasm or nucleus, which was detected in 10 fields of vision under high magnification $(\times 400)$. The interpretation of the results of immunohistochemistry was based on a consensus review by two pathologists.

\section{Follow-up}

The patients were scheduled for follow-up every 1 to 3 months in the first year after tumor resection and at 6 months of intervals thereafter. Thoracic and abdominal multi-detector CT scans were performed at least once a year after surgery. The contrast-enhanced CT examination or biopsy was undertaken when recurrence was suspected during follow-up. If patients were not followed-up at our hospital, we recorded their survival status by telephone as well as the results of their previous examinations carried out in other hospitals.

\section{Statistical analysis}

Categorical data were examined using the $x^{2}$ test and Fisher's exact test. Non-normally distributed continuous variables were examined using Mann-Whitney $U$ test. Disease-free survival (DFS) was calculated as the interval between surgery and first disease recurrence, including 
local-regional recurrence and distant metastasis, or death from any causes. The survival time was compared using the Kaplan-Meier method and log-rank test. Cox proportional hazards model was used to assess the effects of clinical and pathological characteristics on univariate and multivariate analyses of DFS. Multivariate analysis was performed using backward Cox proportional hazards regression with a step-down method. Variables with $P$-values $<0.10$ on univariate analysis were used as input variables for multivariate analysis. The statistical analysis was performed using SPSS 22.0 software (IBM, Armonk, NY, USA), and $P<0.05$ was considered statistically significant.

\section{Results}

\section{Association between patients' clinicopathological characteristics and CT findings}

Table 1 shows the patients' clinical features according to the type of tumor based on CT findings. Among the 68 IMA patients, $41(60.3 \%)$ patients were female, and 27 (39.7\%) patients were male. Solitary-type tumor was diagnosed in 54 patients, and 14 patients had pneumonictype adenocarcinoma. The median age of the patients with solitary-type was 61 years (range, 56-68 years), and that of patients with pneumonic-type adenocarcinoma was 63 years (range, 53.5-70.5 years). There was no significant difference in demographic data. In addition, the majority of IMA patients had no obvious symptoms. Coughing out white sputum was the main symptom found in 18 patients (33.3\%) with solitary-type, and six patients $(42.9 \%)$ with pneumonic-type, however, no significant difference was found $(P=0.726)$. Besides, 50
(73.5\%) tumors were in T1/T2 stage, and 60 (88.2\%) were in N0 stage. Based on the CT findings, $\mathrm{T}$ and $\mathrm{N}$ stages significantly differed between the two types of patients with IMA. The pneumonic-type was associated with a higher pathological stage $(P<0.001)$ and relatively high $\mathrm{T}$ stage and $\mathrm{N}$ stage $(\mathrm{P}<0.001, P=0.008$, respectively). Moreover, in our series, high expression of TTF-1 was found in 32/54 (59.3\%) cases with solitary-type, while only one patient with pneumonic-type showed positive TTF-1 expression $(P=0.001)$. Additionally, positive CK7 expression was identified in 66 cases (Table 2).

Survival analyses between patients with solitary-type and those with pneumonic-type based on CT findings

To investigate the effects of radiological subtype on the survival, we compared the survival rate between the patients with solitary-type (thereafter named solitary group) and those with pneumonic-type (thereafter named pneumonictype group). As a result, the median follow-up time was 10.75 (range, 1.4-89.2) months. The 5-year DFS rate was 64.7 and $0 \%$ for solitary group and pneumonic-type group, respectively. Compared with the solitary group, the pneumonic-type group showed a significantly poorer DFS $(P=0.004)$ (Fig. 3). Furthermore, 9 patients developed cancer recurrence and/or metastases after resection in pneumonic-type group. The initial metastases were intrathoracic metastases, including lung metastasis, pleural dissemination, and mediastinal lymph node metastasis.

Risk factors correlated with postoperative prognosis According to univariate analysis with Cox proportional hazards model for DFS, T stage, pathological stage, TTF-1

Table 1 Clinical characteristics of tumor type based on computed tomography findings

\begin{tabular}{|c|c|c|c|c|}
\hline Characteristics & Total No. (\%) & Solitary type No. & Pneumonic type No. & $P$ value \\
\hline Total of patients & 68 & 54 & 14 & \\
\hline Age & & & & 0.885 \\
\hline Median (interquartile range) & $61(12)$ & $61(12)$ & $63(17)$ & \\
\hline Gender & & & & 0.339 \\
\hline Male & $27(39.7)$ & 23 & 4 & \\
\hline Female & $41(60.3)$ & 31 & 10 & \\
\hline Smoking history & & & & 1.000 \\
\hline Positive & $20(29.4)$ & 16 & 4 & \\
\hline Negative & $48(70.6)$ & 38 & 10 & \\
\hline Symptom & & & & 0.726 \\
\hline Positive & $24(35.3)$ & 18 & 6 & \\
\hline Negative & $44(64.7)$ & 36 & 8 & \\
\hline Type of surgery & & & & 0.229 \\
\hline Wedge resection & $7(10.3)$ & 7 & 0 & \\
\hline Lobectomy & $59(86.8)$ & 46 & 13 & \\
\hline Bilobectomy & $2(2.9)$ & 1 & 1 & \\
\hline
\end{tabular}


Table 2 Pathologic characteristics of tumor type based on computed tomography findings

\begin{tabular}{|c|c|c|c|c|}
\hline Characteristics & Total No. (\%) & Solitary type No. & Pneumonic type No. & $P$ value \\
\hline Total of patients & 68 & 54 & 14 & \\
\hline Pleural visceral invasion & & & & 0.884 \\
\hline Yes & $16(23.5)$ & 12 & 4 & \\
\hline No & $52(76.5)$ & 42 & 10 & \\
\hline T stage & & & & $<0.001$ \\
\hline $\mathrm{T} 1 / \mathrm{T} 2$ & $50(73.5)$ & 48 & 2 & \\
\hline $\mathrm{T} 3 / \mathrm{T} 4$ & $18(26.5)$ & 6 & 12 & \\
\hline N stage & & & & 0.008 \\
\hline NO & $60(88.2)$ & 51 & 9 & \\
\hline N1/N2/N3 & $8(11.8)$ & 3 & 5 & \\
\hline Pathological stage & & & & $<0.001$ \\
\hline |/II & $55(80.9)$ & 50 & 5 & \\
\hline III & $13(19.1)$ & 4 & 9 & \\
\hline TTF-1 expression & & & & 0.001 \\
\hline Positive & $33(48.5)$ & 32 & 1 & \\
\hline Negative & $35(51.5)$ & 22 & 13 & \\
\hline CK-20 expression & & & & 0.189 \\
\hline Positive & $19(27.9)$ & 16 & 3 & \\
\hline Negative & $49(72.1)$ & 38 & 11 & \\
\hline CK 7 expression & & & & 1.000 \\
\hline Positive & $66(97.1)$ & 52 & 14 & \\
\hline Negative & $2(2.9)$ & 2 & 0 & \\
\hline
\end{tabular}

TTF-1 thyroid transcription factor-1, CK cytokeratin

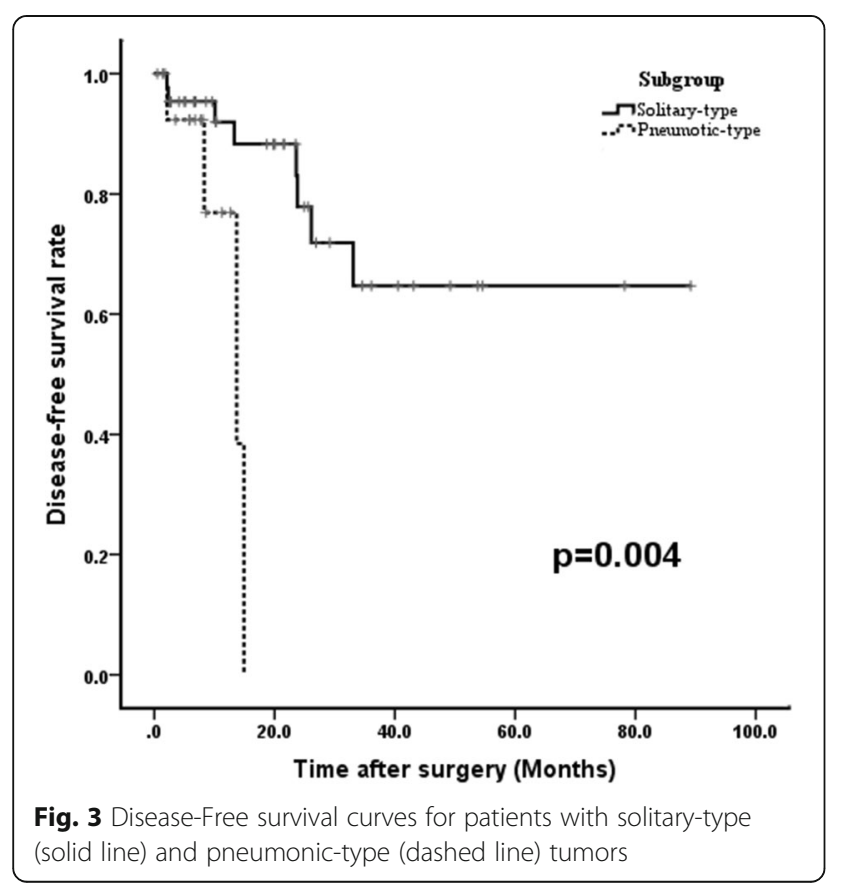

expression, and pneumonic pattern on CT scan were significant factors correlated with DFS $(P=0.014, P=0.013$, $P=0.029, P=0.011$, respectively) (Table 3 ). Multivariate analysis showed that pneumonic-type on CT scan was an independent prognostic factor for DFS [hazard ratio $(\mathrm{HR})=6.764,95 \%$ confidence interval (CI): 1.56329.269, $P=0.011]$, indicating that patients in pneumonic-type group had a worse survival outcome and higher risk for recurrence compared with those in solitary group (Table 3).

\section{Discussion}

The aim of this study was to distinguish the differences between clinicopathological characteristics and prognosis of subtypes of IMA according to CT findings. Based on the 2011 ATS/IASLC/ERS classification system for lung adenocarcinoma, several scholars have already demonstrated that system has a clear prognostic significance. IMAs have been considered to have worse prognosis than lung non-mucinous adenocarcinoma [16, 17]. Several previous cohort studies have indicated that pure IMA have better prognosis compared with mixed mucinous/nonmucinous IMA, and that mixed mucinous/ nonmucinous pattern is an independent risk factor for 
Table 3 Univariate and Multivariate Analyses with Cox Proportional Hazards Model for Disease-free Survival

\begin{tabular}{|c|c|c|c|c|c|c|}
\hline \multirow[t]{2}{*}{ Prognostic factors } & \multicolumn{3}{|c|}{ Univariate analysis } & \multicolumn{3}{|c|}{ Multivariate analysis } \\
\hline & $\mathrm{HR}$ & $95 \% \mathrm{Cl}$ & $P$ value & $\mathrm{HR}$ & $95 \% \mathrm{Cl}$ & $P$ value \\
\hline Age (> 65 vs. $\leq 65)$ & 3.180 & $0.955-10.589$ & 0.059 & $\ldots$ & $\ldots$ & $\ldots$ \\
\hline Gender (Male vs. Female) & 0.618 & $0.199-1.919$ & 0.406 & $\ldots$ & $\ldots$ & $\ldots$ \\
\hline Smoking history (Yes vs. No) & 1.177 & $0.354-3.912$ & 0.790 & $\ldots$ & $\ldots$ & $\ldots$ \\
\hline T stage (T3 4 vs. T1 2) & 5.552 & $1.411-21.842$ & 0.014 & $\ldots$ & $\ldots$ & $\ldots$ \\
\hline N stage (N1 3 vs. N0) & 2.636 & $0.286-24.260$ & 0.392 & $\ldots$ & $\ldots$ & $\ldots$ \\
\hline Pathological stage (III vs.I + II) & 5.467 & $1.428-20.930$ & 0.013 & $\ldots$ & $\ldots$ & $\ldots$ \\
\hline TTF-1 (+vs.-) & 0.239 & $0.066-0.861$ & 0.029 & $\ldots$ & $\ldots$ & $\ldots$ \\
\hline CK20 (+vs.-) & 0.984 & $0.265-3.661$ & 0.981 & $\ldots$ & $\ldots$ & $\ldots$ \\
\hline CT feature, pneumonic type (Yes vs. No) & 6.764 & $1.563-29.269$ & 0.011 & 6.764 & $1.563-29.269$ & 0.011 \\
\hline
\end{tabular}

HR Hazard Ratio, 95\%Cl 95\%Confidence Interval, TTF-1 thyroid transcription factor-1, CK cytokeratin

$P$ value, from Wald Chi-Square Test in Cox Regression

DFS [13, 14]. To date, previous studies have focused on the influence of the pathological classification on prognosis and genetic findings [3, 13], in which the differences between pure IMA subgroups based on CT findings and optimal management for IMA have not been precisely elucidated. Recent researches [10, 11] have indicated that thin-section CT images for IMAs could be helpful for predicting prognosis before surgical resection. Based on CT findings, Shimizu et al. [10] have divided lung IMA into three subtypes, including solid, bubbling, and pneumonic types, and have reported that the pneumonic-type was correlated with a significantly worse outcome compared with solid or bubbling type $(P=0.018)$. Nevertheless, their research cohort included only 29 IMA patients, suggesting that their observations were obviously limited by sample size. Furthermore, Watanabe et al. [11] have reported a series of 40 surgically resected patients with mucinous adenocarcinoma which were divided into two groups (pneumonic-type and solitary) based on the CT scans, with the similar outcome in survival analysis. Nonetheless, 14 out of 40 patients were mucinous AIS or MIA. Both subtypes showed 100\% 5-year survival rate if they were fully resected. Therefore, we can conclude that it is not feasible to accurately assess the differences in clinicopathological features and prognosis of IMA subgroups based on CT scan.

In the current cohort, all patients were diagnosed with pure IMA. The IMAs were classified into two groups, involving pneumonic-type group $(n=14)$ and solitary group $(n=54)$, in which the majority of patients were in stage I/II. Importantly, it was revealed that within $11.8 \%$ of the patients showed lymph node metastasis, and $88.2 \%$ of the patients had nodal stage N0, while there was no case with extrathoracic metastasis. Further statistical analysis showed that pneumonic-type group had a significantly higher rate of lymph node metastasis than solitary group $(P=0.008)$. These data were consistent with several previous studies [12, 18-20]. Tsuta et al. [20] reported that the lymph node-positive rate was $6.8 \%$, which was lower compared with other subtypes of lung adenocarcinoma, such as acinar, papillary, micropapillary, and solid predominant patterns. Because lymph node metastasis is associated with poorer prognosis, it can be used to explain the unfavorable outcome in patients with pneumonic-type adenocarcinoma.

In our cohort, it was revealed that IMA patients were almost older, female (60.3\%), non-smoker (70.6\%), as well as asymptomatic when were initially diagnosed (64.7\%). However, no significant differences in demographic characteristics were observed between types of tumor on CT. These findings were consistent with the results reported by previous studies [11, 12, 19, 21], suggesting that IMA mainly occurs at a greater frequency in female and non-smoking patients. In the majority of cases, it may be difficult to distinguish pneumonia and pneumonic types of IMA based on initial CT findings. In the present study, we found that the excessive coughing with excessive white sputum was more common in patients with pneumonic-type compared with that with solitary-type. These data were consistent with a previous study as well [11]. This might be due to that the pneumonic-type was associated with higher stage and uncontrolled growth of tumor cells, which may cause excessive production of mucus and its discharge through the upper airways. The symptom of coughing with excessive white sputum may help early diagnosis of pneumonic-type adenocarcinoma, while the correlation between symptoms and pathology needs to be further studied.

According to the 8th edition of the TNM staging classification, tumor size is considered as the most important prognostic factor. Tumor being greater than $5 \mathrm{~cm}$, that is defined as T3/4 stage, is taken as a high risk factor into account. In the current study, according to univariate analysis of 68 IMA patients, there was a 
significant worse DFS for T stage (T3 4 versus T1 2), TTF-1 (positive versus negative), pathological stage (III versus I+II) and pneumonic type (Yes versus No). Pneumonic-type showed larger tumor size and more lymph node metastases compared with solitary-type. Therefore, the TNM classification and pathological stage were significantly higher in pneumonic-type group compared with solitary group; besides, pneumonic types had a significantly poorer survival time for DFS. These data were consistent with other studies [10-12]. With reference to poor survival outcome, we found that pneumonic-type was an independent risk factor for poor survival in patients with IMA, while age, $\mathrm{T}$ stage, $\mathrm{N}$ stage, TTF-1, and pathological stage in our study were not independent predictors of DFS on the multivariate analysis. Recently, Lee et al. [12] reported a cohort of IMA patients and found that both tumor size $(\mathrm{HR}=$ 1.370, 95\% CI: $1.141-1.645, P=0.001)$ and $\mathrm{SUV}_{\max }$ $(\mathrm{HR}=1.338,95 \%$ CI: $1.160-1.544, P<0.001)$ were significant independent poor prognostic predictors for DFS, however, the consolidative pattern based on CT features was not related to DFS $(P=0.280)$. This could be due to the TNM stage of their involved IMA patients indicated based on the 7th edition of the TNM classification system and the rate of lymph node metastasis of consolidative type patients was $0 \%$; additionally, different $\mathrm{CT}$ features and clinical relevance of covariates were compared with the present study.

TTF-1 expression is an important prognostic indicator of lung adenocarcinoma. Winslow et al. [22] found that increased expression of TTF-1 suggests a better prognosis, while decreased expression increases tumor colonization and metastatic ability. Previous studies [23, 24] have also demonstrated that TTF-1 positive expression is an important prognostic factor for lung adenocarcinoma. According to previous researches, the majority of the IMAs expressed CK7 ( 88\%) and CK20 ( 54\%) [25-27]. However, the expression of TTF-1 rate is different in the IMA, and its expression rate in IMA is often lower than that in non-mucinous adenocarcinoma [28, 29]. Our data indicated that the DFS was significantly different between IMA patients with positive and negative expressions of TTF-1 $(P=0.029)$. The DFS of patients with negative expression of TTF-1 was worse than those with positive expression. In addition, in this group of cases, the expression of TTF-1 in pneumonia-type IMA was mostly negative, with only one positive case, which may be related to the advanced stage of the pneumonia-type IMA. Zhang et al. [30] reported that negative expression of TTF-1 tends to be more frequent in higher stage tumors, which is consistent with our findings.

The present study has several limitations. Firstly, it was a retrospective study with relatively small sample size. Secondly, only DFS was considered, while overall survival was not calculated, which should be addressed by further studies. Moreover, our survival analysis did not examine the status of gene mutation in all the patients in the present cohort, suggesting that further study of molecular data should be conducted.

\section{Conclusions}

Compared to Pneumonic-type IMA, the Solitary-type IMA is associated with a lower stage and better prognosis. Besides, pneumonic-type is a significant independent risk factor for shorter DFS in patients with IMA.

\section{Abbreviations}

CT: Computed tomography; IASLC/ATS/ERS: International Association for the Study of Lung Cancer/American Thoracic Society/European Respiratory

Society; IMA: Invasive mucinous adenocarcinoma; NSCLC: Non-small cell lung cancer

Acknowledgments

Not applicable.

\section{Authors' contributions}

$\mathrm{KN}, \mathrm{WN}$ and $\mathrm{HY}$ designed this study. KN and YXZ collected data. KN, WN and YXZ wrote this manuscript. HY, WN and KN revised the manuscript. KN, WN and YXZ contributed equally to this paper and were co-first authors. All authors read and approved the final manuscript.

\section{Funding}

This study was founded by the National Natural Science Foundation of China [grant numbers 81671679, 81601988], Shanghai Municipal Science and Technology Development Fund [grant number 15411952000] and the Shanghai Hospital Development Center Foundation [grant number SHDC12014227].

\section{Availability of data and materials}

The datasets analysed during the current study are available from the corresponding author on reasonable request.

\section{Ethics approval and consent to participate}

This retrospective study was approved by the Institutional Review Board of Shanghai Changzheng Hospital (Shanghai, China), and the requirement for informed consent was waived.

Consent for publication

Not applicable.

\section{Competing interests}

The authors declare that they have no competing interests.

\section{Author details}

${ }^{1}$ Department of Imaging and Nuclear Medicine, Changzheng Hospital, Second Military Medical University, Shanghai 200003, China. ${ }^{2}$ Department of Radiology, Shanghai Chest Hospital, Shanghai Jiaotong University, No.241 Huaihai West Road, Xuhui Area, Shanghai 200030, China. ${ }^{3}$ Department of Respiration, Shanghai Chest Hospital, Shanghai Jiaotong University, Shanghai 200030, China. ${ }^{4}$ School of pharmacy, Queen's University Belfast, Medical Biology Centre, 97 Lisburn Road, Belfast BT9 7BL, Northern Ireland, UK.

Received: 26 August 2018 Accepted: 1 July 2019

Published online: 10 July 2019

\section{References}

1. Travis WD, Brambilla E, Noguchi M, Nicholson AG, Geisinger KR, Yatabe Y, et al. International Association for the Study of Lung Cancer/American Thoracic Society/European Respiratory Society international multidisciplinary classification of lung adenocarcinoma. J Thorac Oncol. 2011;6:244-85. 
2. Travis WD, Brambilla E, Noguchi M, Nicholson AG, Geisinger K, Yatabe Y, et al. International Association for the Study of Lung Cancer/American Thoracic Society/European Respiratory Society: international multidisciplinary classification of lung adenocarcinoma: executive summary. Proc Am Thorac Soc. 2011;8:381-5.

3. Shim HS, Kenudson M, Zheng Z, Liebers M, Cha YJ, Hoang Ho Q, et al. Unique genetic and survival characteristics of invasive mucinous adenocarcinoma of the lung. J Thorac Oncol. 2015;10:1156-62.

4. Russell PA, Wainer Z, Wright GM, Daniels M, Conron M, Williams RA. Dose lung adenocarcinoma subtype predict patients survival? A clinicopathologic study based on the new International Association for the Study of Lung Cancer/American Thoracic Society/European Respiratory Society international multidisciplinary lung adenocarcinoma classification. J Thorac Oncol. 2011;9:1496-504.

5. Yoshizawa A, Motoni N, Riely GJ, Sima CS, Gerald WL, Kris MG, et al. Impact of proposed IASLC/ATS/ERS classification of lung adenocarcinoma: prognostic subgroups and implications for further revision of staging based on analysis of 514 stage I cases. Mod Pathol. 2011;(5):653-64.

6. Woo T, Okudera K, Mitsui H, Tajiri M, Yamamoto T, Rino Y, et al. Prognositic value of IASLC/ATS/ERS classification of lung adenocarcinoma in stage I disease of Japanese cases. Pathol Int. 2012;(12):785-91.

7. Cadranel J, Quoix E, Baudrin L, Mourlanette P, Moro-Sibilot D, Morere JF, et al. IFCT-0401 trial: a phase II study of gefitinib administered as first-line treatment in advanced adenocarcinoma with bronchioloalveolar carcinoma subtype. J Thorac Oncol. 2009;4:1126-35.

8. Mansuet-Lupo A, Bobbio A, Blons H, Becht E, Ouakrim H, Didelot A, et al The new histologic classification of lung primary adenocarcinoma subtypes is a reliable prognostic marker and identifies Tumours with different mutation status: the experience of a French cohort. Chest. 2014;146:633-43.

9. Ichinokawa H, Ishii G, Nagai K, Yoshida J, Nishimura M, Hishida T, et al. Clinicopathological characteristics of primary lung adenocarcinoma predominantly composed of goblet cells in surgically resected cases. Pathol Int. 2011;61:423-9.

10. Shimizu K, Okita R, Saisho S, Maeda A, Nojima Y, Nakata M. Clinicopathological and immunohistochemical features of lung invasive mucinous adenocarcinoma based on computed tomography findings. Onco Targets Ther. 2016;10:153-63.

11. Watanabe H, Saito H, Yokose T, Sakuma Y, Murakami S, Kondo T, et al. Relation between thin-section computed tomography and clinical findings of mucinous adenocarcinoma. Ann Thorac Surg. 2015;99:975-81.

12. Lee HY, Cha MJ, Lee KS, Lee HY, Kwon OJ, Choi JY, et al. Prognosis in resected invasive mucinous adenocarcinomas of the lung: related factors and comparison with resected nonmucinous adenocarcinomas. J Thorac Oncol. 2016;11:1064-73.

13. Boland JM, Maleszewski JJ, Wampfler JA, Voss JS, Kipp BR, Yang P, et al. Pulmonary invasive mucinous adenocarcinoma and mixed invasive mucinous/nonmucinous adenocarcinoma-a clinicopathological and molecular genetic study with survival analysis. Hum Pathol. 2018;71:8-19.

14. Luo J, Wang R, Han B, Zhang J, Zhao H, Fang W, et al. Analysis of the clinicopathologic characteristics and prognostic of stage I invasive mucinous adenocarcinoma. J Cancer Res Clin Oncol. 2016;142:1-9.

15. Rami-Porta R, Asamura H, Travis WD, Rusch W. Lung cancer - major changes in the American joint committee on Cancer eighth edition cancer staging manual. CA Cancer J Clin. 2017;67:138-55.

16. Borczuk AC. Prognostic considerations of the new World Health Organization classification of lung adenocarcinoma. Eur Respir Rev. 2016;25:364-71.

17. Van Schil PE, Sihoe AD, Travis WD. Pathologic classification of adenocarcinoma of lung. J Surg Oncol. 2013;108:320-6.

18. Wislez M, Antoine M, Baudrin L, Poulot V, Neuville A, Pradere M, et al. Nonmucinous and mucinous subtypes of adenocarcinoma with bronchioloalveolar carcinoma features differ by biomarker expression and in the response to gefitinib. Lung Cancer. 2010;68:185-91.

19. Lin G, Li H, Kuang J, Tang K, Guo Y, Han A, et al. Acinar-predominant pattern correlates with poorer prognosis in invasive mucinous adenocarcinoma of the lung. Am J Clin Pathol. 2018;149:373-8.

20. Tsuta K, Kawago M, Inoue E, Yoshida A, Takahashi F, Sakurai H, et al. The utility of the proposed IASLC/ATS/ERS lung adenocarcinoma subtypes for disease prognosis and correlation of driver gene alterations. Lung Cancer. 2013;81:371-6.

21. Ichinokawa H, Ishii G, Nagai K, Kawase A, Yoshida J, Nishimura M, et al. Distinct clinicopathologic characteristics of lung mucinous adenocarcinoma with KRAS mutation. Hum Pathol. 2013;44:2636-42.
22. Winslow MM, Dayton TL, Verhaak RG, Kim-Kiselak C, Snyder EL, Feldser DM, et al. Suppression of lung adenocarcinoma progression by Nkx2-1. Nature. 2011;473:101-4.

23. Chung KP, Huang YT, Chang YL, Yu CJ, Yang CH, Chang YC, et al. Clinical significance of thyroid transcription factor-1 in advanced lung adenocarcinoma under epidermal growth factor receptor tyrosine kinase inhibitor treatment. Chest. 2012;141:420-8.

24. Barletta JA, Perner S, lafrate AJ, Yeap BY, Weir BA, Johnson LA, et al. Clinical significance of TTF-1 protein expression and TTF-1 gene amplification in lung adenocarcinoma. J Cell Mol Med. 2009;13:1977-86.

25. Goldstein NS, Thomas M. Mucinous and nonmucinous bronchioloalveolar adenocarcinomas have distinct staining patterns with thyroid transcription factor and cytokeratin 20 antibodies. Am J Clin Pathol. 2001;116:319-25.

26. Lau SK, Desrochers MJ, Luthringer DJ. Expression of thyroid transcription factor-1, cytokeratin 7, and cytokeratin 20 in bronchioloalveolar carcinomas: an immunohistochemical evaluation of 67 cases. Mod Pathol. 2002;15:538-42.

27. Tsuta K, Ishii G, Nitadori J, Murata Y, Kodama T, Nagai K, et al. Comparison of the immunophenotypes of signet-ring cell carcinoma, solid adenocarcinoma with mucin production, and mucinous bronchioloalveolar carcinoma of the lung characterized by the presence of cytoplasmic mucin. J Pathol. 2006:209:78-87.

28. Wu J, Chu PG, Jiang Z, Lau SK. Napsin a expression in primary mucinproducing adenocarcinomas of the lung. an immunohistochemical study Am J Clin Pathol. 2013;139:160-6.

29. Krasinskas AM, Chiosea SI, Pal T, Dacic S. KRAS mutational analysis and immunohistochemical studies can help distinguish pancreatic metastases from primary lung adenocarcinomas. Mod Pathol. 2014;27:262-70.

30. Zhang Y, Wang R, Li Y, Pan Y, Hu H, Zhang Y, et al. Negative thyroid transcription factor 1 expression defines an unfavorable subgroup of lung adenocarcinomas. J Thorac Oncol. 2015:10:1444-50.

\section{Publisher's Note}

Springer Nature remains neutral with regard to jurisdictional claims in published maps and institutional affiliations.
Ready to submit your research? Choose BMC and benefit from:

- fast, convenient online submission

- thorough peer review by experienced researchers in your field

- rapid publication on acceptance

- support for research data, including large and complex data types

- gold Open Access which fosters wider collaboration and increased citations

- maximum visibility for your research: over $100 \mathrm{M}$ website views per year

At $\mathrm{BMC}$, research is always in progress.

Learn more biomedcentral.com/submissions 